agree with this comment, and in our study we had excluded the patients with healed tuberculosis as described in the materials and methods section [1]. The final comment from D. Aggarwal and P.R. Mohapatra is that the duration of treatment is not in accordance with the American Thoracic Society/Infectious Disease Society of America statement [4]. The treatment could have been continued until 1-yr of consecutive sputum culture negativity was established. We had basically followed this recommendation and in our study, the duration of treatment after sputum conversion was $14.2 \pm 3.2$ months. D. Aggarwal and P.R. Mohapatra feel the treatment outcome of a NTM infection should be predicted by evaluating the severity of clinical features, presence of comorbidities, sputum smear grading of acid fast bacilli and radiological findings.

We agree with this comment; however, we think those factors should also be scientifically demonstrated as prognostic markers in future studies.

\section{S. Kuroishi and Y. Nakamura}

Second Division, Dept of Internal Medicine, Hamamatsu University School of Medicine, Shizuoka, Japan.

\section{STATEMENT OF INTEREST}

None declared.

\section{REFERENCES}

1 Kuroishi S, Nakamura Y, Hayakawa H, et al. Mycobacterium avium complex disease: prognostic implication of highresolution computed tomography findings. Eur Respir $J$ 2008; 32: 147-152.

2 Tanaka E, Amitani R, Niimi A, Suzuki K, Murayama T, Kuze F. Yield of computed tomography and bronchoscopy for the diagnosis of Mycobacterium avium complex pulmonary disease. Am J Respir Crit Care Med 1997; 155: 2041-2046.

3 Jeong YJ, Lee KS, Koh WJ, Han J, Kim TS, Kwon OJ. Nontuberculous mycobacterial pulmonary infection in immunocompetent patients: comparison of thin-section CT and histopathologic findings. Radiology 2004; 231: 880-886.

4 Griffith DE, Aksamit T, Brown-Elliott BA, et al. An official ATS/IDSA statement: diagnosis, treatment, and prevention of nontuberculous mycobacterial diseases. Am J Respir Crit Care Med 2007; 175: 367-416.

\title{
COPD and osteoporosis: a lot of work to be done
}

\section{To the Editors:}

There is no doubt that on reading what is suggested by FABBRI $e t$ al. [1] on the view of chronic obstructive pulmonary disease (COPD), a holistic vision of the patient turns out to be extremely absorbing and a cornerstone in our medical grounding.

However, as often happens, reality far exceeds expectations. In a retrospective analysis carried out on 885 outpatients (507 males and 378 females) treated at our pneumatological outpatient clinic over the last 2 yrs, 297 (178 males and 119 females) had COPD; of whom only $10(1.13 \%)$ were diagnosed as suffering from osteoporosis. In all other cases, the necessary examination to assess the mineral bone density was not carried out, in spite of a chronic respiratory pathology and an apparent reduction of muscular mass, owing to reduction in physical exertion, and notwithstanding the steroid therapy. The number of subjects diagnosed is thus a dramatically low percentage, despite the abundance of literature now available. According to the guidelines of the Italian Health Department on osteoporosis [2], prevention of fractures is an objective aimed at improving the quality of life, assisting drastic reduction in social costs and obtaining health economy. With a view to gaining valid prevention of osteoporosis at all ages, the key steps to follow could be the following: stick to a diet rich in calcium and vitamin D; exert physical exercise proportionate to body weight; define mineral bone density; and undergo the correct therapy [3-6]. Aside from our 10 outpatients treated for osteoporosis, no preventive strategy was planned for the remaining patients, nor any stratification of the risk of loss of bone mass was executed. Our conclusions are valid for the sampled patients, but we cannot say whether they can be extended to all patients with COPD. In our view, it is crystalclear that physicians must be aware of the effects on bone mass related to COPD, as well as those of the chronic administration of steroids or due to consequent inaction or smoking. We cannot believe that "the fact that chronic diseases seldom occur in isolation is still largely ignored" [1]. Nevertheless, this analysis proves the lack of preventive intervention in osteoporosis. A reason for this situation could be the economic drift of our public health system and, as a result, the consequent goal is to reach a balanced budget, rather than attain the planned final outcome, irrespective of the self-evident collateral effects and long-term relapses.

To the public health system, the cost per patient for 1-yr of treatment with biphosphonate ranges between $€ 292.00$ and $€ 524.00$ [7], which is not a minor burden, when we consider the number of patients with COPD and of all plausible cases of osteoporosis [8].

Moreover, these data suggest that it is necessary for the daily practical clinic to take into account how important the stratification of the risk of secondary osteoporosis is for all patients with chronic pathologies under steroid therapy, as well as the importance of the preventive nonpharmacological treatment.

Furthermore, based upon scientific evidence, more frequent communication becomes mandatory between medical teams on one hand and those who manage the funds on the other, as far as intentions and objectives are concerned.

\section{S. Zeminian, M. Pattarello and F. Rubini}

Rehabilitative Respiratory Unit, Marzana Hospital, Verona, Italy. 
STATEMENT OF INTEREST

None declared.

\section{REFERENCES}

1 Fabbri LM, Luppi F, Benghè B, Rabe K. Complex chronic comorbidities of COPD. Eur Respir J 2008; 31: 204-212.

2 Italian Health Department. Guidelines on osteoporosis. www.ministerosalute.it/alimenti/nutrizione/linee.jsp?lang= italiano\&label $=$ oste\&id $=83$ Date last updated and accessed: July 3, 2008.

3 Consensus Development Conference. Diagnosis, prophylaxis, and treatment of osteoporosis. Am J Med 1993; 94: 646-650.
4 Consensus Development Statement. Who are candidates for prevention and treatment for osteoporosis? Osteop Int 1997; 7: $1-6$.

5 Osteoporosis prevention, diagnosis, and therapy. NIH Consensus Statement 2000; 17: 1-45.

6 Italian Society for Osteoporosis and Mineral Metabolism and Bone Disease (SIOMMMS). Guidelines for diagnosis, prevention and therapy of osteoporosis. Edn EDIMES, Pavia, 2006.

7 The Medical Letter, Italian Edition 2008:1;1.

8 Hubbard R, Tattersfield A, Smith C, West J, Smeeth L, Fletcher A. Use of inhaled corticosteroids and the risk of fracture. Chest 2006; 130: 1082-1088.

DOI: $10.1183 / 09031936.00034608$

\section{Doubts about the role of cannabis in causing lung}

\section{cancer}

\section{To the Editors:}

We read with interest the article by ALDINGTON et al. [1] entitled "Cannabis use and risk of lung cancer: a case-control study". However, we are concerned that the conclusion stating that "long-term cannabis use increases the risk of lung cancer in young adults" is inadequately supported by the data, given the study's several methodological flaws.

First, ALDINGTON et al. [1] argue that the risk of lung cancer increases linearly by $8 \%$ per pack-yr smoked, based on the assumption that cancer risk from cannabis smoking is similar to cancer risk from tobacco smoking, which also increases linearly with pack-yrs. However, their own data show that the cancer risk from tobacco smoking does not increase linearly, but rather geometrically, rendering the assumption invalid. Furthermore, the authors found an increased risk of lung cancer only in the highest tertile of cannabis smokers, rendering the posited linear relationship inappropriate.

Secondly, since over half of the subjects were $>50$ yrs of age, it seems misleading to refer to them as "young adults".

Thirdly, although the study is powered at $80 \%$ to detect an odds ratio (OR) of 2.4 for lung cancer in the $15 \%$ of the population who smoke cannabis, it is only the considerably smaller population that had smoked $>10.5$ joint-yrs that showed an increased risk. Epidemiological data on the percentage of the population that has smoked $>10.5$ joint-yrs are not available, but if the sample provided by ALDINGTON et al. [1] is representative, the figure is only $4.5 \%$. Therefore, the study is underpowered at $25 \%$ for an OR of 2.4 and $31 \%$ for an OR of 2.7. A sample of 12,000 cases and 50,000 controls would be required to detect, at $80 \%$ power, a relative risk of 1.08 per joint-yr reported by the authors. Consequently, their conclusion is probably only a chance finding.

Fourthly, although the study is termed "case-control", the cases are adequately matched with controls only for sex. Subjects in the case group were disproportionate in age, education, wealth, heritage and smoking history; all of which are independent risk factors for lung cancer.

With that in mind, ALDINGTON et al. [1] dismissal of a much larger study of 2,252 subjects by HASHIBE et al. [2] in 2006, which found no increase in cancer risk, even in those patients who smoked $>60$ joint-yrs on the grounds that controls were matched for neighbourhood is puzzling, because controls should be matched for neighbourhood.

Finally, an observational study such as ALDINGTON et al. [1] cannot establish causality, only correlation. Even presupposing that the study was methodologically impeccable, the strongest conclusion that could be drawn would be that "long-term cannabis use is associated with an increased risk of lung cancer", not that cannabis use causes lung cancer.

Given the acrimonious debate over the role of cannabis in society, and the substantial chance that this paper will contribute to public policy decisions, we feel it is important to keep conclusions that are stated in abstracts and papers as close to the actual scientific findings as possible.

\section{R.A. Sewell*\#, A.J. Cohn ${ }^{\Uparrow}$ and M.C. Chawarski ${ }^{\#}$}

*VA Connecticut Healthcare, West Haven, "Yale University School of Medicine, New Haven, CT, and "Advisory Committee on Medical Marijuana, Portland, OR, USA. 